\title{
Src tyrosine kinase inhibits apoptosis through the Erk1/2- dependent degradation of the death accelerator Bik
}

\author{
J Lopez ${ }^{1,2}$, C Hesling ${ }^{1}$, J Prudent ${ }^{1}$, N Popgeorgiev ${ }^{1}$, R Gadet ${ }^{1}$, I Mikaelian ${ }^{1}$, R Rimokh ${ }^{1}$, G Gillet ${ }^{\star, 1}$ and P Gonzalo ${ }^{\star, 1,2}$
}

Src, the canonical member of the non-receptor family of tyrosine kinases, is deregulated in numerous cancers, including colon and breast cancers. In addition to its effects on cell proliferation and motility, Src is often considered as an inhibitor of apoptosis, although this remains controversial. Thus, whether the ability of Src to generate malignancies relies on an intrinsic aptitude to inhibit apoptosis or requires preexistent resistance to apoptosis remains somewhat elusive. Here, using mouse fibroblasts transformed with v-Src as a model, we show that the observed Src-dependent resistance to cell death relies on Src ability to inhibit the mitochondrial pathway of apoptosis by specifically increasing the degradation rate of the BH3-only protein Bik. This effect relies on the activation of the Ras-Raf-Mek1/2-Erk1/2 pathway, and on the phosphorylation of Bik on Thr124, driving Bik ubiquitylation on Lys33 and subsequent degradation by the proteasome. Importantly, in a set of human cancer cells with Src-, Kras- or BRAF-dependent activation of Erk1/2, resistances to staurosporine or thapsigargin were also shown to depend on Bik degradation rate via a similar mechanism. These results suggest that Bik could be a rate-limiting factor for apoptosis induction of tumor cells exhibiting deregulated Erk1/2 signaling, which may provide new opportunities for cancer therapies.

Cell Death and Differentiation (2012) 19, 1459-1469; doi:10.1038/cdd.2012.21; published online 2 March 2012

p60 ${ }^{\mathrm{c}-\mathrm{src}}$ is the founding member of the Src family of tyrosine kinases. Within the cell, these kinases act on a multiplicity of pathways that regulate proliferation, survival, motility and adhesion. At the molecular level, a variety of mechanisms are involved, including phosphorylation of numerous substrates on tyrosyl residues, as well as activation of the Ras/mitogenactivated protein kinase (MAPK), PI3K/Akt and STAT3dependent signaling pathways. ${ }^{1}$ The viral isoforms, including v-Src, of Src-like kinases exhibit high transforming capacities. $^{2}$ In humans, $c$-src is deregulated in $>80 \%$ of colon cancers, and may be critical for tumor progression. ${ }^{3}$ Src levels are also high in many other cancers. Importantly, Src is activated in a number of tumors resistant to current therapies and might be a target of major therapeutic interest. ${ }^{4}$

Src, as a number of proteins with oncogenic potential such as Myc, E2F-1, Ras or c-Jun, can induce the paradoxical outcomes of cell multiplication, senescence or apoptosis, depending on the cellular context. ${ }^{5-8}$ Apoptosis activation by Src seems to rely on several mechanisms; Src can stimulate c-myc expression, ${ }^{9}$ leading to the activation of p19-ARF and $\mathrm{p} 53 ;{ }^{10}$ it may also depend on E2F-1. ${ }^{11}$ On the other hand, a number of reports support the opposing view that Src could inhibit apoptosis by downregulating proapoptotic genes ${ }^{12}$ or upregulating anti-apoptotic genes. ${ }^{13-16}$ In addition, Src is a potent activator of the PI3K/Akt pathway, which protects against pro-apoptotic stimuli via the phosphorylation and inactivation of death accelerators such as Bad, Bax and caspase-9. ${ }^{17-19}$ Src-dependent activation of p38-MAPK similarly leads to the phosphorylation of caspases-8 and -3 . $^{20}$

However, from these reports, it was unclear whether or not the ability of Src to generate malignancies in mammals required preexistent resistance to cell death, and therefore depended on a cooperation between oncogenes and antiapoptotic proteins as observed for Myc and Bcl-2. Another possibility was that Src might also directly inhibit apoptosis, as observed in avian cells. ${ }^{13}$ Here, on the basis of experiments performed in both whole cells and isolated mitochondria, we report that Src-transformation increased resistance of mouse fibroblasts to cell death as the direct consequence of the accelerated degradation of the pro-apoptotic protein Bik by the proteasome. Importantly, this mechanism resulting from the phosphorylation of Bik by the extracellular signal-related kinase (Erk) $1 / 2$ kinase was also shown to occur in human cancer cells with deregulated Erk1/2 activity, possibly opening up new therapeutic avenues.

\footnotetext{
${ }^{1}$ Centre de Recherche en Cancérologie de Lyon, Université Lyon 1 - Inserm U1052 - CNRS UMR5286, Centre Léon Bérard, Bâtiment Cheney D, 28 rue Laënnec, Lyon 69373 Cedex 08, France and ${ }^{2}$ Hospices Civils de Lyon, Fédération de Biochimie Nord, Hôpital de la Croix-Rousse, 103, grande rue de la Croix-Rousse, Lyon F-69317 Cedex 04, France

${ }^{*}$ Corresponding authors: G Gillet or P Gonzalo, Centre de Recherche en Cancérologie de Lyon, Centre Léon Bérard, Université de Lyon - Inserm 1052 - CNRS 5286 , Bâtiment Cheney D, 28 rue Laënnec, Lyon 69373 Cedex 08, France. Tel: + 334691666 56; Fax: + 334691666 60; E-mail: germain.gillet@ lyon.unicancer.fr (GG) or Tel: + 334691666 10; Fax: + 334787827 20; E-mail: philippe.gonzalo@lyon.unicancer.fr (PG) Keywords: apoptosis; Bik; Src; proteasome; Erk1/2; Bim

Abbreviations: MAPK, mitogen-activated protein kinase; Erk, extracellular signal-related kinase; ER, endoplasmic reticulum; DMK, double mutant on lysine Received 11.7.11; revised 17.1.12; accepted 30.1.12; Edited by SJ Martin; published online 02.3.12
} 


\section{Results}

\section{v-src-transformed NIH-3T3 cells are apoptosis} resistant. 3T3 cells transformed by a temperature-sensitive v-Src mutant were used to study the effects of Src expression on apoptosis sensitivity in mammalian cells. We found that $v$ src-transformed 3 T3 cells (but not cells grown at $39.5^{\circ} \mathrm{C}$ ) were strongly resistant to a variety of apoptosis inducers such as UV, etoposide, staurosporine and thapsigargin (Figure 1a and Supplementary Figure S1A). To gain further insight into the mechanisms underlying this resistance to apoptosis, we assessed the concentration of recombinant tBid required to release cyt-c from isolated mitochondria. ${ }^{21}$ This tBid concentration was much higher when using mitochondria from $v$-src-transformed cells $(20 \mathrm{nM})$ than from non-transformed cells $(7.5 \mathrm{nM})$, an effect that disappeared when mitochondria came from $v$-src-transformed cells grown at $39.5^{\circ} \mathrm{C}$ (Figure $1 \mathrm{~b}$ and Supplementary Figure S1B).

Src increases the threshold for Bax activation. Above results suggested that Src might inhibit the Bax/Bak activation cascade. This was checked both in cellulo and in vitro. Bax translocation and activation on staurosporine treatment were observed in control cells but not in $v$-srctransformed cells (Supplementary Figure S1C). In vitro, incubation with $10 \mathrm{nM}$ tBid for 15 min triggered Bax insertion in control mitochondria but not in mitochondria isolated from $v$-src-transformed cells (Supplementary Figure S1D), although tBid bound normally to these mitochondria (Supplementary Figure S1E) and tBid-induced cyt-c release depended on Bax activation (Supplementary Figure S1F).

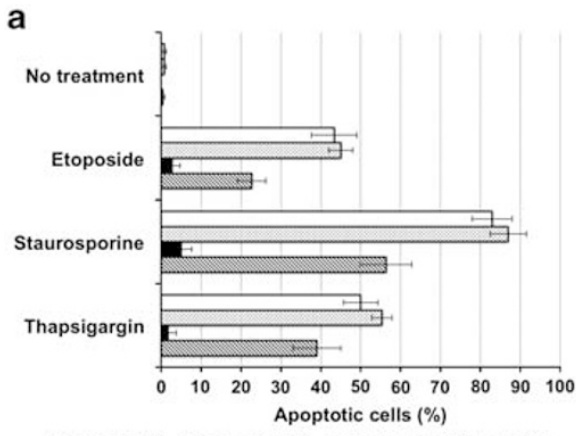

aControl $37^{\circ} \mathrm{C}$ aControl $39.5^{\circ} \mathrm{C}$ avSrc $37^{\circ} \mathrm{C}$ avSrc $39.5^{\circ} \mathrm{C}$

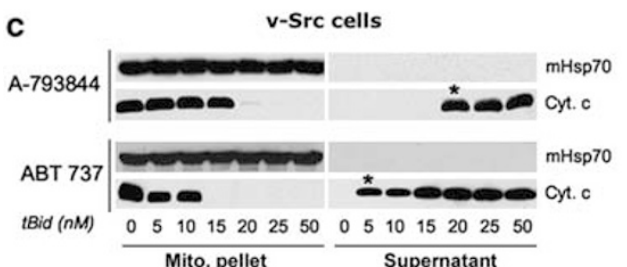

b

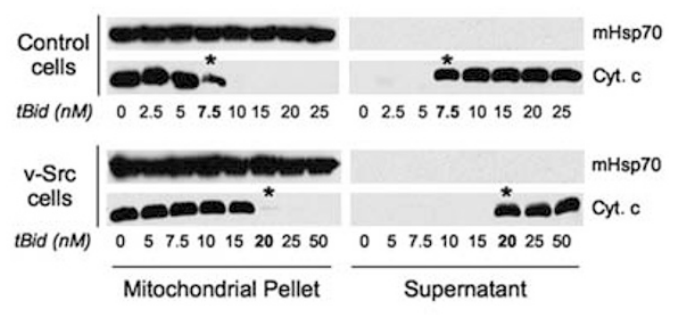

d
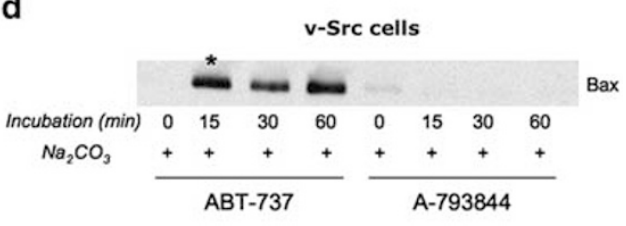

Figure 1 Src activity induces resistance to apoptosis by inhibiting the cyt-c release machinery. (a) Non-transformed cells (control $37^{\circ} \mathrm{C}$ and control $39.5^{\circ} \mathrm{C}$ ) and $v$-srctransformed cells with either active $\left(\mathrm{v}-\operatorname{Src} 37^{\circ} \mathrm{C}\right)$, or heat inactivated $\operatorname{Src}\left(\mathrm{v}-\operatorname{Src} 39.5^{\circ} \mathrm{C}\right)$ were treated with different apoptosis inducers. Percentages of apoptotic cells (mean \pm S.D. from three independent experiments) are shown. This experiment indicates that resistance to apoptosis of transformed cells depended on Src activity. (b) Isolated mitochondria from control and $v$-src-transformed cells were incubated with increasing concentrations of tBid for 30 min and recovered by centrifugation. Cyt-c release was revealed by western blotting on mitochondria pellets and supernatants. Mitochondrial Hsp70 was used as a loading control. The required tBid concentration threshold for cyt-c release (*) was 2.7-fold higher in v-Src mitochondria. (c) Cyt-c release from isolated mitochondria from v-Src cells was performed as in (b) except that $1 \mu \mathrm{M}$ of either ABT-737 or A-793844 was added during incubation. As shown ( $\left.{ }^{*}\right)$, ABT-737, contrary to its inactive enantiomer A-793844, lowers by four the tBid concentration required to release cyt-c. (d) Isolated mitochondria from v-Src cells incubated with $10 \mathrm{nM} \mathrm{tBid} \mathrm{and} 1 \mu \mathrm{M}$ of either ABT-737 or A-793844 were submitted to alkali treatment and analyzed by anti-Bax immunoblotting. ABT-737, but not A-793844, restores Bax insertion

Figure 2 Bik controls apoptosis induction of 3 T3 cells. (a) Western blotting analyses of the Bcl-2 family proteins in control (Ctrl) and v-src-transformed cells (Src). Differential centrifugation was used for cell fractionation. Each experiment was repeated at least twice. Mitochondrial $\mathrm{Hsp} 70$ and $\alpha$-tubulin were used as loading controls for mitochondrial fractions and $10000 \times g$ supernatants $(10000 \mathrm{~g} \mathrm{SN}$ ), respectively (loading $100 \mu \mathrm{g}$ proteins). Whole cell lysates from HeLa (Bcl-w), BP3 (Bfl1/A1), 293T (Noxa and Bmf) and WT MEF (Bak) cells were used as positive controls when proteins were undetected. (b) Apoptosis of untransformed 3 T3 cells (Ctrl cells) on staurosporine, thapsigargin and etoposide treatment was compared after knockdown by shRNA of BH3-only proteins Bik, Bim, Bad and Puma. A scrambled sequence was used as a control (shCTRL). Apoptosis of untransformed 3 T3 cells induced by thapsigargin or staurosporine depends significantly on Bik levels (mean \pm S.D. in three independent experiments). Knockdown efficacies are shown in S2B. (c) Contributions of the BH3-only proteins expressed in $3 \mathrm{~T} 3$ cells to the release of cyt-c were studied by incubating mitochondria from non-transformed cells silenced for bik, bad, bim or puma with increasing tBid concentrations. The tBid concentration allowing cyt-c release $\left({ }^{*}\right)$ decreased significantly when bik, but not bad, bim, puma, was silenced. (d) v-src-transformed (Src) and non-transformed cells (Ctrl) were transfected with Bik and/or Bcl-XL expression vectors when indicated and grown in soft agar. The effect of Src inhibition by dasatinib (dasa) is also shown. On day 20, cells were examined under bright field illumination for colony formation $(\times 4)$. Colonies from triplicate wells were counted after crystal violet staining. (e) Apoptosis of $v$-src-transformed cells silenced for either bik, bad, bim or puma and treated overnight with dasatinib (Dasa) was induced by staurosporine, thapsigargin or etoposide. Percentages of apoptotic cells was reduced significantly on staurosporine and thapsigargin treatment when bik was silenced while the death-inducing effect of etoposide was compromised on silencing of puma (mean \pm S.D. from three independent experiments) 
In addition, ABT-737, an inhibitor of anti-apoptotic Bcl-2 proteins, ${ }^{22}$ dramatically reduced tBid concentration required for cyt-c release and Bax activation (Figures 1c and d). These experiments showed that the Bax-dependent cyt-c release machinery was functional in $v$-src-transformed cells. However, the threshold of apoptosis activation was significantly raised compared with non-transformed cells.
The decrease in Bik protein levels is responsible for the increased resistance to apoptosis of src-transformed 3T3 cells. As Bcl-2 family proteins are main regulators of Bax activation, we analyzed the levels of these proteins in transformed and non-transformed cells (Figure 2a). Bcl-2, $\mathrm{Bcl}-\mathrm{X}_{\mathrm{L}}$ and $\mathrm{Mcl}-1$ were the only anti-apoptotic proteins to be detected. $\mathrm{Bcl}-\mathrm{X}_{\mathrm{L}}$ and $\mathrm{Mcl}-1$ concentrations were not a

mHsp70

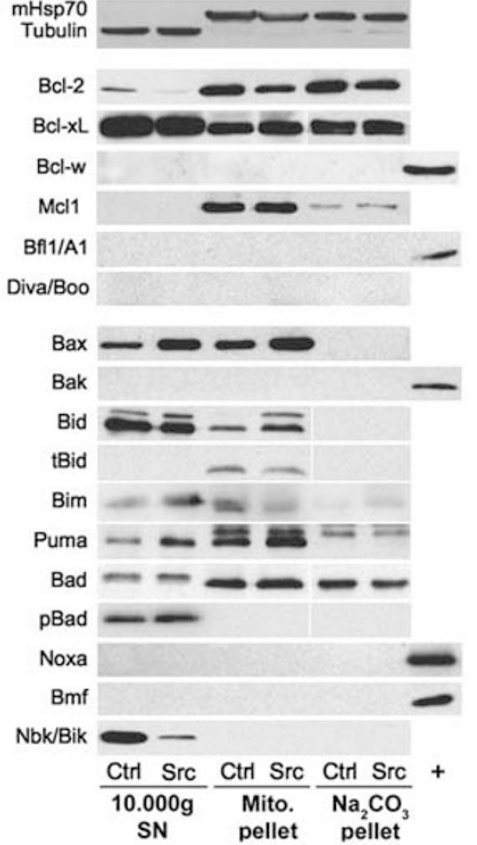

C

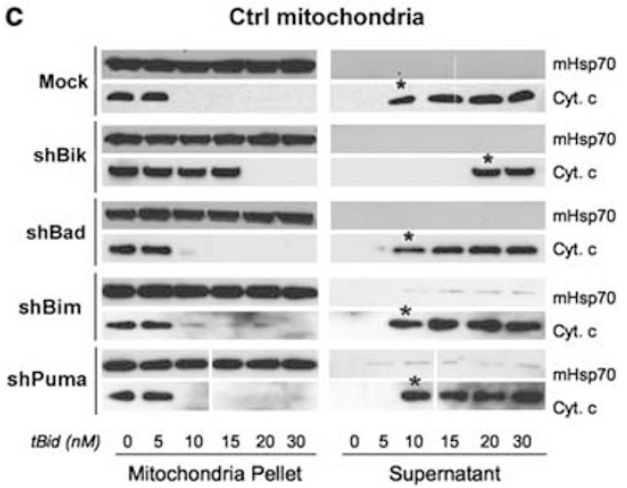

b

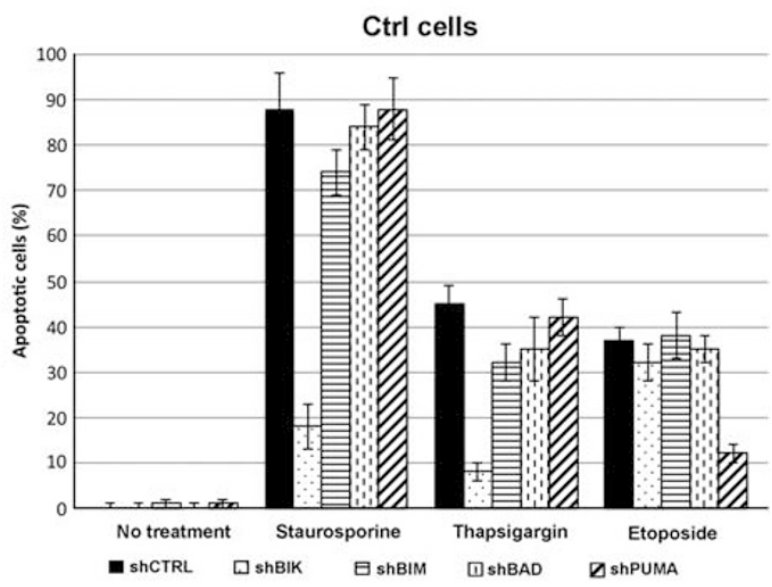

d
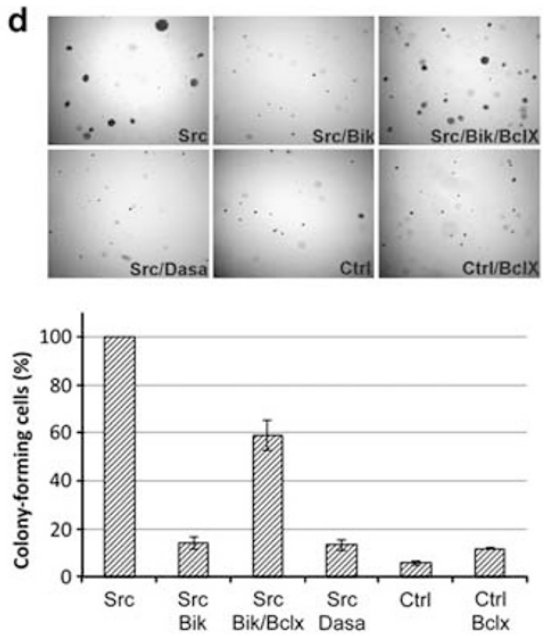

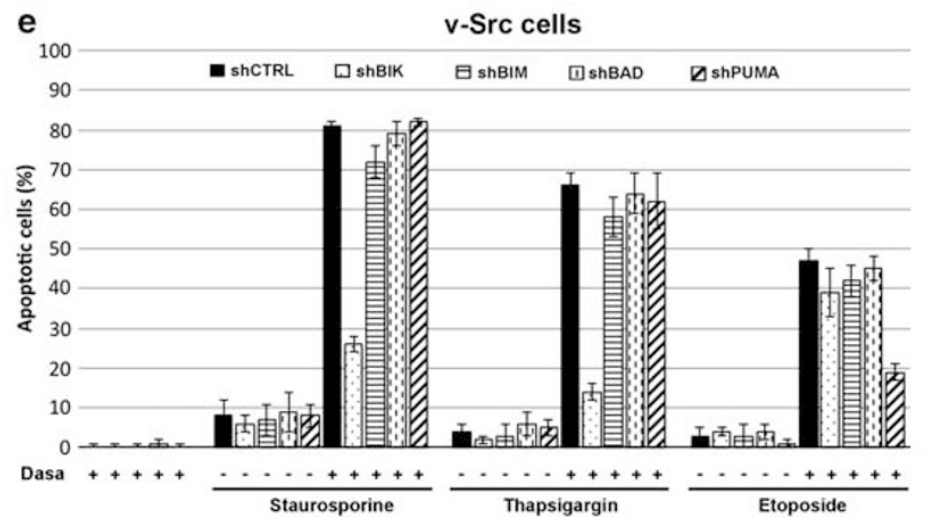


significantly altered by $v$-src expression whereas Bcl-2 levels were slightly decreased. Thus, resistance to apoptosis of $v$-src-transformed cells was not the result of an accumulation of anti-apoptotic proteins.

Regarding pro-apoptotic proteins, Bax, but not Bak, could be detected. In v-src-transformed cells, Bax was more abundant but not activated (Supplementary Figure S1D, left two first lanes). Among the BH3-only subclass, Bid, Bim, Puma, Bad and Bik were detected. Bid and Puma levels were very similar in both cells. Bad levels were also similar, and, as expected, phosphorylated Bad was cytosolic while non-phosphorylated Bad was mitochondrial. ${ }^{18}$ Low levels of Bim were detected, mainly at the mitochondria in untransformed cells and in the $10000 \times g$ supernatant for $v$-src-transformed cells, a band-shift being present between both forms. In contrast, in $v$-src-transformed cells, Bik protein levels dropped dramatically in the $10000 \times g$ supernatant, consistent with Bik preferred location in the endoplasmic reticulum $(\mathrm{ER}){ }^{23}$

Two series of experiments confirmed the importance of Bik levels for apoptosis control in 3T3 cells. First, we observed that apoptosis of non-transformed cells, which exhibited high Bik protein levels, was specifically decreased on bik silencing on thapsigargin and staurosporine induction in contrast to bim, puma or bad silencing. Of note, etoposide-induced apoptosis depended on Puma (Figure 2b). Moreover, isolated mitochondria from bik-silenced cells required $20 \mathrm{nM}$ tBid to release cyt-c, but only $10 \mathrm{nM}$ tBid in bad-, bim- or puma-silenced cells (Figure 2c). In addition, cyt-c release could be prevented through preincubation with an anti-Bik antibody, but not with an anti-Bad antibody (Supplementary Figure S2A). Altogether, these results highlighted the pivotal role of Bik in apoptosis induction in untransformed cells.

A second series of experiments demonstrated that apoptosis resistance of $v$-src-transformed cells depended on Bik protein drop. (i) Bik overexpression in $v$-src-transformed cells resulted in apoptosis in response to staurosporine (Supplementary Figure S2C). (ii) The forced accumulation of Bik in $\mathrm{v}$-src-transformed cells prevented the formation of colonies in soft agar, which seemed to depend on Bik pro-apoptotic activity because co-transfection with $\mathrm{Bcl}-\mathrm{X}_{\mathrm{L}}$ restored this typical property of Src transformation (Figure 2d). (iii) The selective Src kinase family inhibitor dasatinib restored normal Bik protein levels and apoptosis but failed to do so when bik, but not bad, bim or puma, was silenced (Figure 2e).
Src induces Bik ubiquitylation and subsequent proteasomal degradation. First, we showed that Src activity was responsible for decreased endogenous Bik levels. Indeed, endogenous Bik levels were decreased on ectopic expression of WT c-Src or active Y527F in 3T3 cells and, conversely, Bik accumulated on Src kinase inhibition by temperature shift, herbimycin or dasatinib in $v$-srctransformed cells (Figure $3 a$ ). We then investigated how Src controlled Bik protein levels in 3T3 cells. No decrease in Bik mRNA levels was observed in $v$-src-transformed cells (Figure $3 b$ ), suggesting a post-transcriptional mechanism. Therefore, we measured HA-tagged Bik protein half-life in cells expressing or not activated c-Src (Y527F) or v-Src (Figure 3c). Remarkably, Bik protein half-life, close to $3 \mathrm{~h}$ in control cells, dropped to 45 min when Src activity was turnedon. Moreover, Src inhibition by herbimycin or dasatinib restored Bik protein stability. These results suggested that Src stimulated Bik protein degradation, which resulted in much lower Bik protein levels in $v$-src-transformed cells in steady-state conditions. Inhibition of the proteasome with MG132 increased HA-Bik and endogenous Bik protein stability in $v$-src-transformed cells (Figures $3 c$ and d) and restored staurosporine-induced apoptosis, which was counteracted by bik but not bim knockdown (Figure 3e).

Immunoprecipitation of HA-tagged Bik from $v$-src-transformed cells treated with MG132 allowed the detection of a main specific band of about $60 \mathrm{kDa}$, which was revealed by both anti-Bik and anti-ubiquitin antibodies and that disappeared on bik silencing (Figure $3 \mathrm{f}$ ). In cell lysates from cells co-expressing ubiquitin-myc and HA-Bik, a similar band of $60 \mathrm{kDa}$ was also revealed with anti-HA antibody following immunoprecipitation with anti-myc antibody, indicating that Bik is polyubiquitylated (Figure $3 \mathrm{~g}$ ). The observed size of the band suggested that it might correspond to a penta- or hexaubiquitylated form of Bik.

Bik K33R mutant (but not Bik K13R) as well as the double (K13R/K33R) mutant referred to as double mutant on lysine (DMK) were stabilized in $v$-src-transformed cells as compared with WT-Bik (Figure 3c). As shown by further immunoprecipitation experiments, Bik ubiquitylation disappeared with herbimycin and with the Bik K33R and DMK mutants whereas the Bik K13R mutant was still ubiquitylated (Figure $3 \mathrm{~g}$ ). Altogether, these results suggested that Src enhanced Bik polyubiquitylation on lysine 33 and subsequent proteasomal degradation.

Figure 3 Src induces the ubiquitylation of Bik and subsequent proteasomal degradation. (a) Endogenous Bik level was compared with Src level and Src kinase activity as monitored by Y416 phosphorylation. 3T3 cells were transfected with either wild-type (WT) or activated (Y527F) c-Src and, v-src-transformed $3 T 3$ cells had v-Src kinase activity inhibited either by herbimycin, dasatinib or by incubation at $39.5^{\circ} \mathrm{C}$. These results indicated that Bik levels and Src activities were inversely related. (b) Relative expression $(\mathrm{RQ})$ of bik was quantified by RT-QPCR in control and v-Src cells in two independent experiments. (c) Bik protein half-life was measured in control cells, control cells transfected with activated c-Src (Y527F) and v-src-transformed cells. Cells were transfected with wild-type (WT) HA-Bik or the different HA-Bik K $>$ R mutants for $24 \mathrm{~h}$ following treatment with herbimycin, dasatinib or MG132 when indicated. Then, cells were incubated with cycloheximide and actinomycin D for the indicated times. Bik degradation rates were assessed by anti-HA-tag western blotting in corresponding whole cell lysates. (d) Endogenous Bik expression was compared by western blotting in control and v-Src cells treated or not with MG132. (e) Apoptosis of v-src-transformed cells silenced for either bik or bim and treated or not with MG132 was induced by staurosporine. Proteasome inhibition greatly resensitized $v$-src-transformed cells, which was counteracted when bik but not bim was silenced. Results are mean of two independent experiments. (f) $v$-srctransformed 3T3 cells were transfected with myc-tagged ubiquitin and wild-type HA-Bik and shBik when indicated. Cells were then grown with MG132 (excepted for first lane). Immunoprecipitation was performed with an anti-HA antibody and revealed by immunostaining with either anti-Bik or anti-ubiquitin antibodies. Specific bands accumulating on proteasome inhibition and immunostained for Bik and ubiquitin are indicated $\left(^{*}\right)$. Arrows point to the IgG light and heavy chains. (g) $v$-src-transformed 3 T3 cells expressing myc-tagged ubiquitin were grown with MG132 after being transfected with either WT HA-Bik, HA-Bik K > R mutants at position 13 (K13R), 33 (K33R) or both (DMK), or with an empty vector. Immunoprecipitation was performed with an anti-myc antibody and revealed by immunostaining with an anti-HA antibody. The arrow points to the $60 \mathrm{kDa}$ immunoreactive band corresponding to a polyubiquitylated form of Bik, which was absent on herbimycin treatment or when K33 was mutated. The $55 \mathrm{kDa}$ band corresponds to the anti-myc IgG heavy chain. Bik was used as a loading control 
Src accelerates Bik degradation via the Ras-Raf-Mek1/2Erk1/2 pathway. Next, we focused on the signaling pathway connecting Src to Bik degradation. Using Bik degradation rate as a read-out, we screened inhibitors of a variety of kinases involved in Src signaling. Among the transduction pathways expected to be stimulated by $v$-src-transformation, PI3K, Mek1/2, p38-MAPK, JNK and mTor pathways were activated (Supplementary Figures S3A and S3C). However, the only compounds leading to Bik stabilization were the Mek1/2/5 inhibitor UO126 and the more specific Mek1/2 inhibitor PD184352 suggesting that only the Ras-Raf-Mek1/2-Erk1/2 pathway was involved in the enhanced degradation of Bik (Figure 4a). Consistent with the involvement of this pathway, Bik stabilization was observed in v-src-transformed cells following Ras inhibition by the RasN17 dominant-negative mutant or following c-Raf, Mek1/2 or Erk1/2 inhibitions with

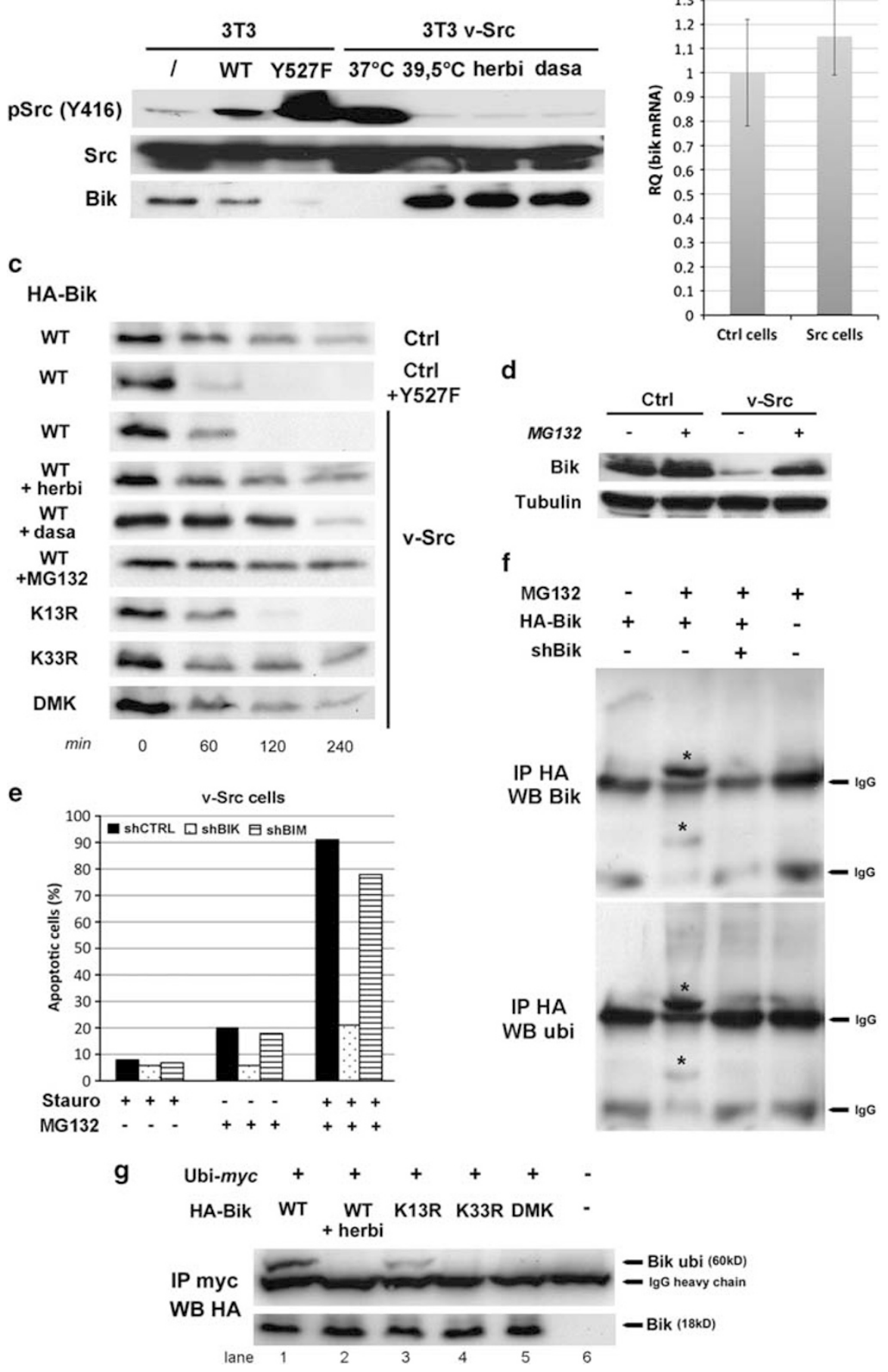


a

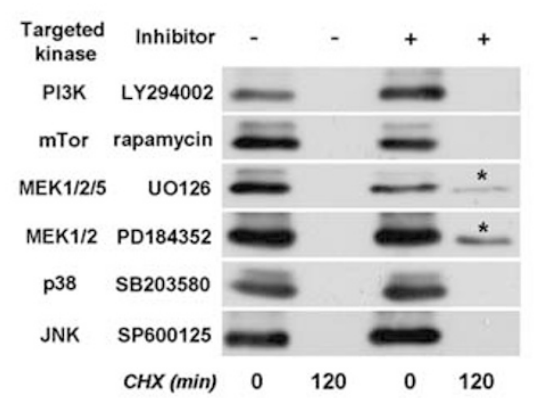

C

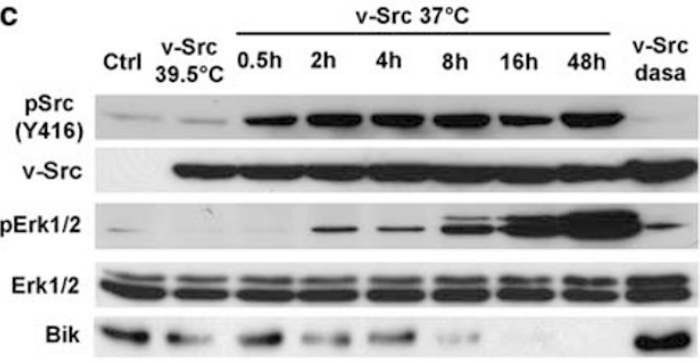

b

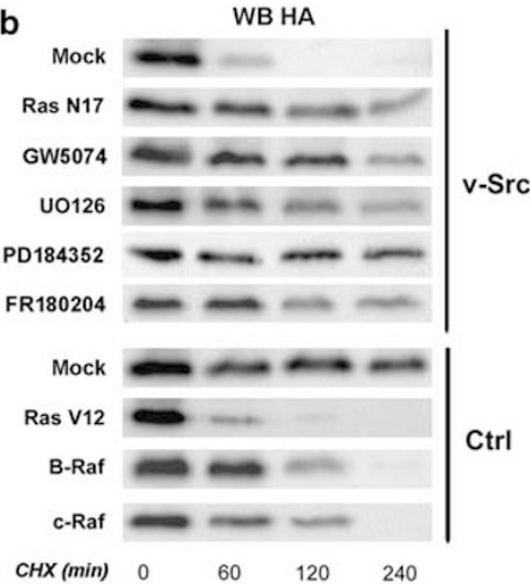

$\begin{array}{lllll}\text { CHX (min) } & 0 & 60 & 120 & 240\end{array}$

d

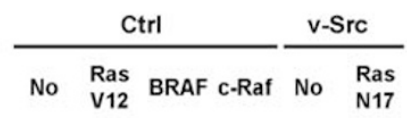

Tubulin

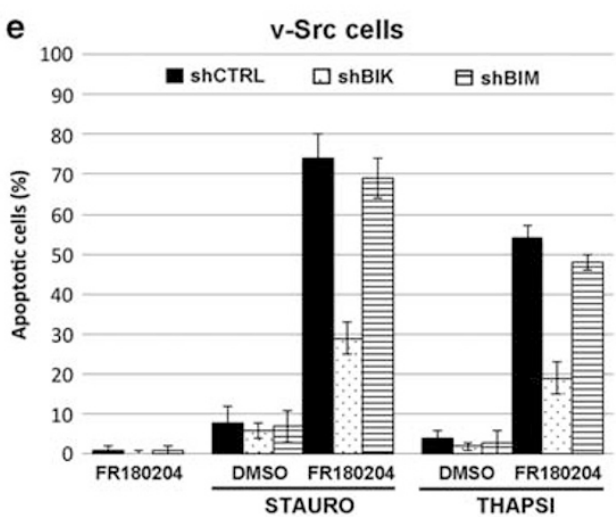

Figure $4 \mathrm{Src}$ accelerates Bik degradation via the Ras-Raf-Mek1/2-Erk1/2 pathway. (a) A pharmacological screen was used to identify the pathway mediating the Src-induced degradation of Bik. HA-Bik degradation rate was assessed as in Figure $3 \mathrm{c}$ in $v$-src-transformed cells treated with various kinase inhibitors. Only the Mek1/2 inhibitors increased Bik stability (*). Control of the actual activity of these pathways in v-src-transformed cells and efficacy of these inhibitors is shown in Supplementary Figures S3A and S3C. (b) Bik protein half-life was measured as in (a): inhibitors of the Ras-Raf-Mek1/2-Erk1/2 pathway were used in v-src-transformed cells (N17: dominant-negative Ras; GW5074: c-Raf inhibitor; UO126: Mek1/2/5 inhibitor; PD184352: more specific Mek1/2 inhibitor; FR180204: Erk1/2 inhibitor). Conversely, this pathway was activated in untransformed cells by expression of RasV12, B-RAF and c-Raf; the control of expression of these activators is shown in Supplementary Figure S3B. Activation of the Ras pathway accelerated Bik degradation, whereas its inhibition increased Bik stability. (c) Src kinase activation (pY416) began $0.5 \mathrm{~h}$ after shifting v-src-transformed cells from restrictive $\left(39.5^{\circ} \mathrm{C}\right)$ to permissive temperature $\left(37^{\circ} \mathrm{C}\right)$; Erk1/2 activation (p-Erk1/2 controlled with total Erk1/2) and endogenous Bik degradation both began at $2 \mathrm{~h}$. (d) Endogenous Bik levels in untransformed cells expressing RasV12, BRAF or c-Raf and in v-src-transformed cells transfected with RasN17. (e) Apoptosis on staurosporine or thapsigargin induction of $v$-src-transformed cells silenced for either bik or bim and treated or not with the Erk1/2 inhibitor FR180204. Erk1/2 inhibition restores normal apoptosis in v-src-transformed cells in bim- but not in bik-knockdown cells

pharmacological agents (Figure 4b, Supplementary Figures S3A and S3C). Reciprocally, overexpression of constitutively active RasV12, as well as B-RAF or c-Raf, increased Bik degradation rates in non-transformed cells (Figure $4 \mathrm{~b}$, Supplementary Figures S3B and S3C).

When $v$-src-transformed cells were shifted from restrictive to permissive temperature, Src kinase reactivation was detected as early as $30 \mathrm{~min}$. As early as $2 \mathrm{~h}$, Erk $1 / 2$ activation was observed, together with the decrease in endogenous Bik levels (Figure 4c). This experiment showed that Bik degradation was indeed under Src control through Erk1/2 activation.
Bik degradation on Erk1/2 activation and Bik accumulation on Erk1/2 inhibition were also demonstrated on endogenous Bik (Figure 4d). Importantly, Bik accumulation on Erk $1 / 2$ inhibition correlated with increased apoptosis of $v$-src-transformed cells on thapsigargin or staurosporine induction. The critical role of Bik was further demonstrated by the fact that bik but not bim knockdown counteracted resensitization to death stimuli by the Erk1/2 inhibitor FR180402 (Figure 4e).

Bik is a direct target of the Erk1/2 kinase. Next, we studied how Erk1/2 activation could lead to Bik degradation. 
2D-electrophoreses were performed on extracts of transformed or non-transformed cells, both overexpressing HA-Bik. HA-Bik appeared as a single spot in control cells (isoelectric point pl 6.7), whereas a 'multispot chain' was observed in $v$-src-transformed cells, with apparent pls of the additional spots corresponding to the calculated pls of mono-, di- and tri-phosphorylated protein (Figure $5 \mathrm{a}$ ). These results suggested that Bik might be phosphorylated in $v$-srctransformed cells.

It had been previously described that human Bik and pErk1/2 interacted with one another, leading to $p$-Erk1/2 sequestration in the cytosol. ${ }^{24}$ Here, we postulated that p-Erk1/2 was able to phosphorylate and drive Bik degradation. Endogenously expressed Bik was co-immunoprecipitated using a total Erk1/2 antibody (Figure 5b). The absence of interaction when Erk1/2 activation was prevented by PD184352 (whereas this interaction was detected when Erk1/2 was activated) strongly suggested that only p-Erk1/2 might interact with Bik. As expected, the L63G Bik mutant corresponding to the human L61G Bik mutant was unable to interact with $p$-Erk1/2 anymore (Figure $5 c$ ).

To identify Bik phosporylated residues, the four putative Erk1/2 phosphorylation sites (Ser or Thr in S/T.P motif) were mutated into either alanine (non-phosphorylable) or aspartate (phosphomimetic) and consequences of these mutations on Bik stability were investigated. In $v$-src-transformed cells, compared with WT HA-Bik and to the S42A, S76A, S130A mutants, the T124A mutant was stabilized, (Figure $5 \mathrm{~d}$ ). Conversely, the phosphomimetic T124D mutant showed a shortened half-life in control cells. Interestingly, the mouse L63G Bik mutant unable to interact with $p$-Erk1/2 also exhibited a longer half-life in $v$-src-transformed cells. Of note, the T124A and T124D mutants both interacted with p-Erk1/2 (Figure 5c). These results suggested that $p-E r k 1 / 2$ phosphorylated Bik T124, which led to Bik accelerated degradation.

To confirm that Bik was not phosphorylated by a downstream kinase, we performed direct in vitro kinase assays with pure p-Erk2 kinase and WT, T124A or L63G HA-Bik immunopurified from 293T cells overexpressing the p35 caspase inhibitor. Cells were grown with PD184352 to inhibit Bik endogenous phosphorylation and MG132 to promote Bik accumulation (Figure 5e). Bik phosphorylation, detected with a phospho-Thr antibody, was revealed when both p-Erk2 and WT HA-Bik were present, while it could not be detected for the T124A and L63G mutants or in the absence of p-Erk2. In addition, HA-Bik was phosphorylated in cellulo on a threonine residue in $v$-src-transformed cells, and this phosphorylation was no longer observed on PD184352 treatment or for the L63G mutant (Figure 5f).

Altogether, these results show that activated Erk1/2 docked at the N-terminus of the BH3-domain of Bik leading to phosphorylation the conserved $\mathrm{T} 124$ residue. This appeared to be the initial event leading to Bik degradation.

Bik T124 phosphorylation targets Bik for ubiquitylation. Finally, we investigated whether Bik ubiquitylation depended on T124 phosphorylation (Figure $5 \mathrm{~g}$ ). Pull-down experiments of $m y c$-tagged ubiquitin were performed as in Figure $3 \mathrm{~g}$. In $v$-src-transformed cells, the $60 \mathrm{kDa}$ polyubiquitylated form of Bik remained undetectable when $\mathrm{p}$-Erk1/2 was inhibited or with the L63G and T124A mutants. In control cells, Bik ubiquitylation was detected with the T124D Bik mutant or after activation of the Ras-Raf-Mek1/2-Erk1/2 pathway by RasV12. These results showed that Bik T124 phosphorylation by $p$-Erk $1 / 2$ triggered Bik ubiquitylation.

The Src-Ras-Raf-Mek1/2-Erk1/2 pathway also accelerates Bik degradation in human cancer cells by phosphorylating the $\mathbf{S 1 2 4}$ residue. To investigate whether Bik stability and apoptosis were controlled in a similar manner in human cells, we used a set of four human cancer cells with deregulated Erk1/2 activity and described as overexpressing c-Src: one lung cancer cell line ( $\mathrm{NCl}-\mathrm{H} 460)$ and three colon carcinoma cell lines (LS174T, COLO205 and HCT116). COLO205, LS174T and NCl-H460 exhibited high Src activity (Figure 6a). However, in COLO205 cells, full inhibition of Erk1/2 phosphorylation on PD184352 treatment compared with partial inhibition on dasatinib confirmed that $B R A F^{25}$ and not $c-S r c$, was the driver oncogene. In HCT116 cells, Erk1/2 activation depended on Kras and not on $c$-Src. ${ }^{26} \mathrm{NCl}-\mathrm{H} 460$ cells harbored a Kras mutation, ${ }^{27}$ but $\mathrm{c}-\mathrm{Src}$ was the driver oncogene as shown by the inhibition of Erk1/2 activation on dasatinib treatment. Finally, LS174T cells that harbored an heterozygous Kras mutation $^{28}$ depended mainly on c-Src deregulation for Erk1/2 activation (Figure 6a).

Then, the effects of Src or Mek1/2 inhibitions were tested on Bik or BimEL accumulation (Figure 6a) and apoptosis as assessed by the use of shRNAs targeting Bik, Bim or both (Figures 6b and c, Supplementary Figures S4C-S4E). BimEL was analyzed because it is phosphorylated by p-Erk1/2, which inhibits its interaction with Bax and promotes survival. ${ }^{29}$

In $\mathrm{NCl}-\mathrm{H} 460$ cells, in which Src drove Erk1/2 activation, both dasatinib and PD184352 restored high Bik levels and led to a band-shift described as unphosphorylated BimEL. ${ }^{30}$ However, BimEL levels were low and apoptosis restored by Src or Mek1/2 inhibitors depended mainly on Bik, as in 3T3 cells. In COLO205 cells, in which BRAF drove Erk1/2 activation, Bik accumulation and Bim dephosphorylation were observed on Mek1/2 or BRAF (PLX-4720) inhibitions but not Src inhibition. Accordingly, apoptosis was restored by PD184352 or PLX-4720 but not by dasatinib and depended on Bik, Bim or both proteins (Supplementary Figure S4B for PLX-4720). In HCT116 cells, in which Erk1/2 activation depended on Kras, Mek1/2 inhibition increased Bik levels, inhibited BimEL phosphorylation and restored apoptosis in a Bik or Bim-dependent manner. Finally, in LS174T cells, dasatinib and PD184352 failed to restore normal Bik levels led to unphosphorylated BimEL accumulation and restored apoptosis. Actually, bik mRNA was not expressed in these cells (Supplementary Figure S4A).

Whatever the mechanism, Bik protein was maintained at low levels in the four human cancer cell lines. In addition, these results suggested a conserved pathway for the regulation of Bik protein stability by Erk1/2. This was confirmed by comparing the molecular mechanisms underlying Bik degradation in NCl-H460 and HCT116 cells. 
a
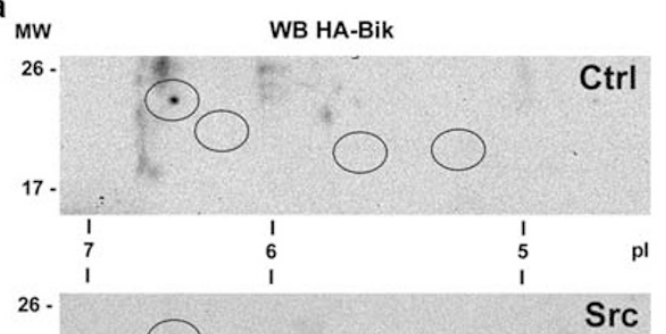

17 .
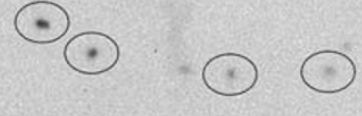

C

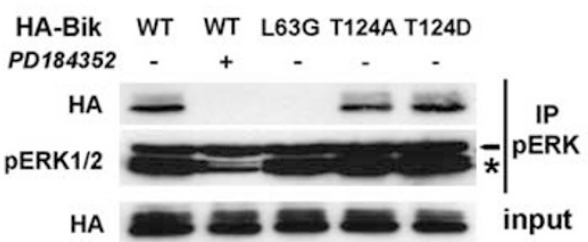

e

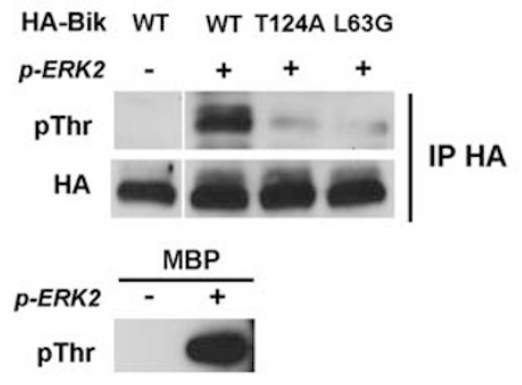

g Ubi- $m$ $\frac{\text { v-Src cells }}{+\quad+\quad+\quad+}$ HA-Bik

f

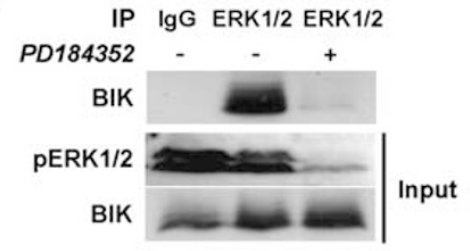

d

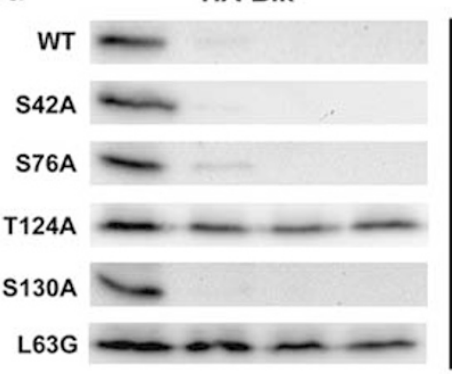

WT

T124D

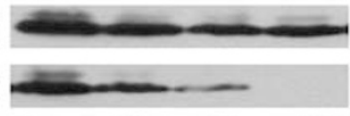

Ctrl

cells

v-Src

cells

$\min$

0

60

$120 \quad 240$

$\begin{array}{lll} & \text { v-Src } & \text { Ctrl } \\ n & \text { HA-Bik } & \text { WT L63G WT }\end{array}$

PD184352

pThr

HA

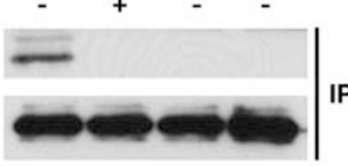

IP HA

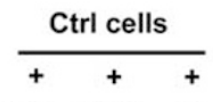

WT T124D WT + RasV12

\section{IP myc}

WB HA

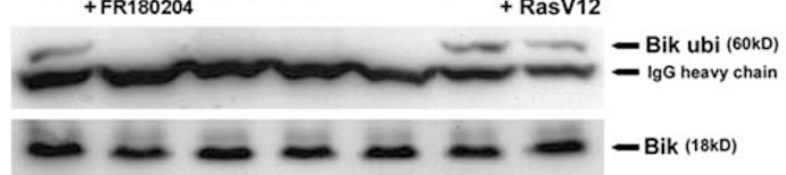

Figure $5 \mathrm{p}$-Erk1/2 directly phosphorylates Bik on T124, which triggers Bik ubiquitylation and degradation. (a) Whole cell lysates of untransformed (Ctrl) and v-srctransformed cells expressing HA-Bik were analyzed by immunostaining using anti-HA antibody after 2-D gel electrophoresis. A unique spot (pl 6.7) was revealed in

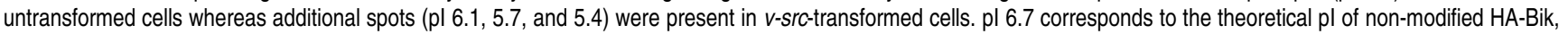
whereas the more acidic pls correspond to mono, bi and tri-phosphorylated HA-Bik. (b) $v$-src-transformed cells were transfected with the p35 caspase inhibitor and treated with MG132 to allow endogenous Bik accumulation. When indicated PD184352 was used to block Erk1/2 activation. Total Erk1/2 was immunoprecipitated from lysates (1:50) and co-immunoprecipitated Bik was revealed by western-blotting. Rabbit lgG were used as negative control when indicated. (c) $v$-src-transformed cells were transfected with wild-type (WT) HA-Bik or the L63G mutant unable to interact with p-Erk1/2 ${ }^{24}$ or phosphomimetic (T124D) or unphosphorylable (T124A) mutant at position 124. p-Erk1/2 was immunoprecipitated from these cells and interaction with Bik was revealed by anti-HA western-blotting. Bik but not the L63G mutant interacted with $p$-Erk1/2. (d) The stability of mutants of putative phosphorylation sites by p-Erk1/2 (S/T.P motif) was measured as in Figure $3 b$. Unphosphorylable Bik mutants (S/T >A) at positions $42,76,124$ and 130 and the L63G mutant were expressed in v-src-transformed cells. Only the T124A and L63G mutants exhibited increased stability in v-src-transformed cells. Phosphomimetic mutants at the above positions were also expressed in control cells: only the T124D mutant displayed here exhibited decreased stability in control cells. (e) p-Erk1/2 in vitro kinase assay. WT, T124A or L63G HA-Bik mutants and the p35 caspase inhibitor were overexpressed in 293T cells grown with MG132 to allow Bik accumulation and PD184352 to inhibit Bik endogenous phosphorylation. Bik, immunopurified with $\mathrm{HA}$-antibodies, was incubated for $1 \mathrm{~h}$ at $37^{\circ} \mathrm{C}$ with $100 \mathrm{U}$ of $\mathrm{p}$-Erk2 and phosphorylation was revealed by anti-phospho-threonine western blotting. MBP phosphorylation was used as a positive control and the absence of phosphorylation of WT Bik in the absence of p-Erk2 was used as a negative control. p-Erk2 directly phosphorylated Bik and T124A and L63G mutations abolished this phosphorylation. (f) Bik endogenous phosphorylation by $p$-Erk1/2 was investigated: WT- and L63G HA-Bik were immunoprecipitated from $v$-src-transformed cells or untransformed cells and analyzed by anti-phospho-threonine western blotting. Bik is phosphorylated on Thr in v-src-transformed cells but not in 3 T3 cells or when Erk1/2 activation or p-Erk1/2 interaction with L63G-Bik is prevented. (g) Ubiquitylation of Bik mutants on the T124 phosphorylation site was investigated as described in Figure 3g. Bik ubiquitylation was only detected in conditions allowing or mimicking Bik T124 phosphorylation 
a

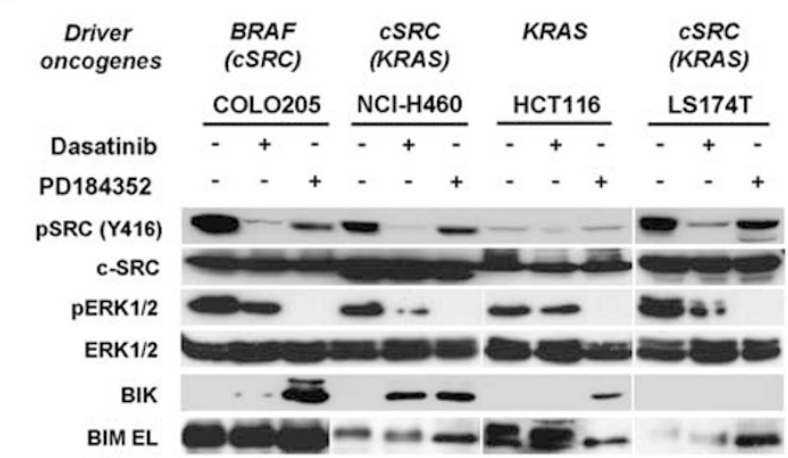

C

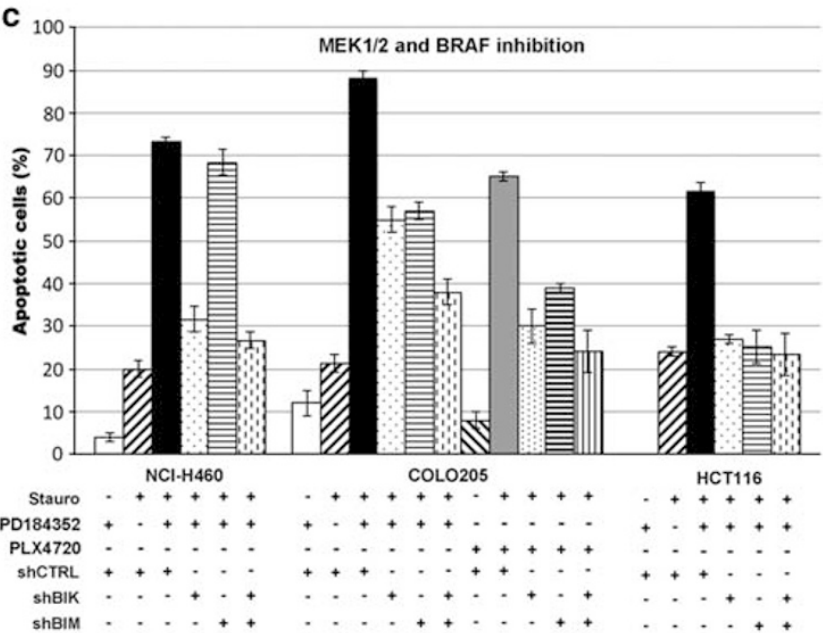

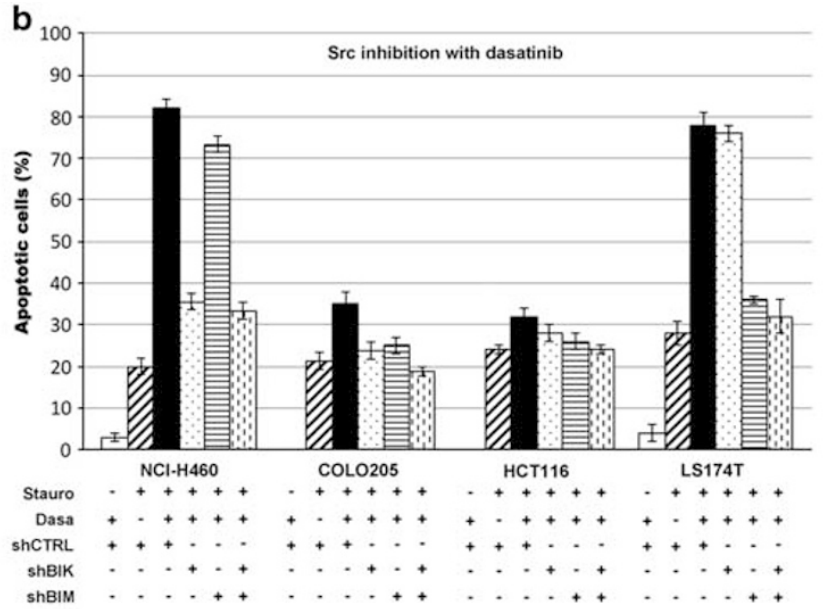

d

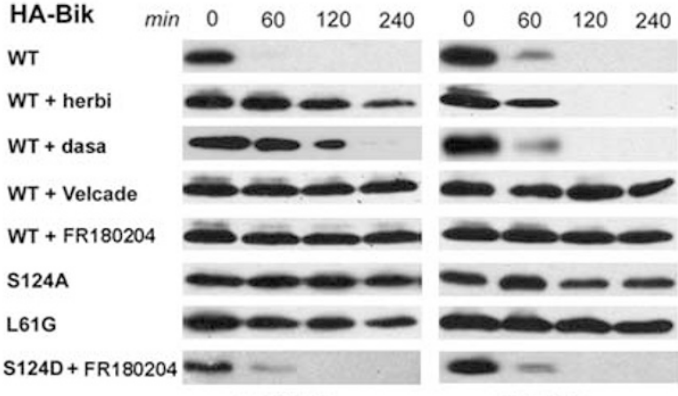

$\mathrm{NCl}-\mathrm{H} 460$

HCT116

Figure 6 Bik degradation is similarly regulated in an Erk1/2-dependent manner in human cancer cell lines (a) The human lung cancer cell line NCl-H460 and human colon carcinoma cell lines COLO205, HCT116 and LS174T were incubated with either vehicle (-), Src inhibitor dasatinib or Mek1/2 inhibitor PD184352 to assess the contribution of Src activity on Erk1/2 activation and endogenous Bik and Bim EL expression. Western blots were carried out on cell lysates to assess Src (pSrc Y416) and Erk1/2 (pErk1/2) activation and Bik and Bim EL expression. (b) Apoptosis was triggered by staurosporine for $6 \mathrm{~h}$ in NCl-H460, COLO205, HCT116 and LS174T cells silenced for bik or/and bim and grown with dasatinib when indicated. Efficacies of the shRNAs used to downregulate endogenous Bik or Bim in the four human cancer cell lines is shown in Supplementary Figure S4C. Apoptosis was restored by dasatinib in NCI-H460 and LS174T cells and depended respectively on Bik and Bim. (c) Apoptosis was triggered as in (b) but on PD184352 treatment. Mek1/2 inhibition restored sensitivity to apoptosis in NCl-H460 where it depended on Bik and in COLO205 and HCT116 where it depended on both Bik and Bim. In COLO205, BRAF inhibition by PLX4720 was similarly able to resensitize cells to apoptosis. Supplementary Figures S4D and S4E are similar to Figures $6 \mathrm{~b}$ and c, except that apoptosis was triggered by thapsigargin. Percentage of apoptotic untreated cells (without apoptosis inducer or kinase inhibitors) was below $2 \%$. (d) $\mathrm{NCl}$ H460 and HCT116 cells were transfected with the indicated HA-Bik constructs and treated with herbimycin, dasatinib, the Erk1/2 inhibitor FR180204 or Velcade, or co-transfected with RasN17. Bik degradation rates were assessed as in Figure 3b. These results show that phosphorylation of human Bik on the S124 residue by p-Erk1/2 stimulates Bik degradation

Three human Bik mutants were generated: the phosphomimetic (S124D) and unphosphorylable (S124A) mutants of the conserved phosphorylation site and the L61G mutant, unable to interact with $\mathrm{p}$-Erk1/2. ${ }^{24} \mathrm{~S} 124 \mathrm{~A}$ and L61G mutations restored Bik stability as well as Erk1/2 inhibition by FR180204 (Figure 6d). Moreover, FR180204 failed to stabilize the S124D mutant, showing that phosphorylation of S124 by $\mathrm{p}$-Erk1/2 was the signal triggering Bik degradation. Proteasome inhibition by bortezomib (Velcade) led to Bik protein stabilization. As expected, Src inhibition led to Bik stabilization in $\mathrm{NCl}-\mathrm{H} 460$ but not in HCT116 cells.

Altogether, these results point to a pivotal role played by Bik in the induction of apoptosis induced by staurosporine and thapsigargin in transformed human cells with deregulated Erk1/2 signaling.

\section{Discussion}

Bik, a suspected tumor suppressor, as a target of Src in mouse and human cancer cells. In this study, we show that, in both mouse and human cells, Src enhances resistance to a number of death stimuli by accelerating the degradation of Bik, the first BH3-only protein to be identified. ${ }^{31}$ Bik is able to trigger typical apoptotic events such as Bax oligomerization, cyt-c release from mitochondria, caspase 3 activation, chromatin condensation and degradation. These results are consistent with the hypothesis that bik might be a tumor suppressor. ${ }^{23}$ Indeed, loss of bik gene expression has been observed in renal cell carcinoma and bik is deleted in $22 \%$ of colorectal cancer cases. 
Erk1/2 drives Bik to degradation by the proteasome. A limited number of instances described the involvement of the proteasome in the control of Bik turnover. ${ }^{32-34}$ However, so far, the upstream regulatory mechanisms have remained elusive. Here, we demonstrate that Src triggers Bik phosphorylation via the Ras-Raf-Mek1/2-Erk1/2 cascade, which targets Bik for ubiquitylation and proteasomal degradation. Moreover, our data indicate that this mechanism is conserved in human cancer cells. Of note, the four human cancer cell lines used in this study exhibit Bik deficiency. Although bik gene expression is turned-off in LS174T cells, the drop in Bik protein levels results from Erk $1 / 2$ activation as a consequence of Kras constitutive activation in HCT116 cells, BRAF mutation in COLO205 and c-Src deregulation in $\mathrm{NCl}-\mathrm{H} 460 .{ }^{26}$ Of note, although the contribution of Bik downregulation to the appearance of chemoresistance was previously suggested, ${ }^{23}$ no connection with Src or Erk1/2 activation had been established to date.

Interestingly, the Bim BH3-only protein was similarly reported to be regulated by $p$-Erk $1 / 2$. In our hands, the two mechanisms of BimEL inhibition by $p$-Erk $1 / 2$ were observed. In COLO205, NCl-H460, HCT116 and v-src-transformed 3T3 cells, the $p$-Erk1/2-dependent phosphorylation of BimEL led to a band-shift already described ${ }^{30}$ that correlated with its inactivation. ${ }^{29}$ In LS174T, the p-Erk1/2-dependent phosphorylation of BimEL led to its degradation. ${ }^{35}$

Dependence of both Bim and Bik on Erk1/2 activity led us to compare their respective contributions with apoptosis induction in human cell lines. In LS174T cells in which bik gene expression is turned-off, resistance to apoptosis depended on BimEL phosphorylation by $\mathrm{p}$-Erk $1 / 2$ whereas in $\mathrm{NCl}-\mathrm{H} 460$ in which BimEL expression is comparatively low, apoptosis resistance depended mainly on the $\mathrm{p}$-Erk $1 / 2$ driven degradation of Bik. Interestingly, in the two other cell lines (COLO205 and HCT116), both active BimEL and Bik levels were restored on inhibition of Erk1/2 activation, while either Bim or Bik knockdown restored resistance to apoptosis.

Thus, as Erk $1 / 2$ activation may result from the deregulation of numerous oncogenes such as Src, Kras, Nras, BRAF, EGFR, BCR-ABL, to which tumor cells can be 'addicted', ${ }^{36}$ the restoration of Bik or Bim protein levels by inhibiting Erk1/2 activation might be a therapeutic avenue in a number cancers with poor prognoses (Figure 7).

\section{Materials and Methods}

Cell culture. NIH-3T3 cell lines were cultured in Dulbecco's modified essential medium supplemented with $10 \%$ fetal bovine serum (FBS), penicillin (100 U/ml) and streptomycin $(100 \mu \mathrm{g} / \mathrm{ml})$. Control and $v$-src overexpressing stable cell lines were established as previously described using a pLNCX plasmid. ${ }^{37} \mathrm{NCl}-\mathrm{H} 460$, COLO205, LS174T and HCT116 cell lines were maintained in RPMI-1640 supplemented with $10 \% \mathrm{FBS}$, penicillin and streptomycin.

Apoptosis induction and assay. Cells were treated with either staurosporine ( $2 \mu \mathrm{M}$ for $8 \mathrm{~h})$, etoposide $(200 \mu \mathrm{M}$, overnight), thapsigargin $(5 \mu \mathrm{M}$, overnight) or UV irradiated (15s, 15-h incubation). Then, apoptosis was assessed by the percentage of apoptotic cells with pyknotic nuclei and diffuse cyt-c staining after cyt-c immunostaining (mAb 6H2, R\&D Systems (Lille, France), 1: 1000).

Pharmacological inhibitors. Drugs were from VWR (Pessac, France): herbimycin $(1 \mu \mathrm{M})$, MG132 $(10 \mu \mathrm{M})$, bortezomib $(1 \mu \mathrm{M})$, LY294002 $(50 \mu \mathrm{M})$, rapamycin $(500 \mathrm{nM})$, UO126 $(5 \mu \mathrm{M})$, SB203580 $(50 \mu \mathrm{M})$, SP600125 (JNK inhibitor II; $5 \mu \mathrm{M})$, FR184352 $(5 \mu \mathrm{M})$ and GW5074 $(5 \mu \mathrm{M})$; from Euromedex

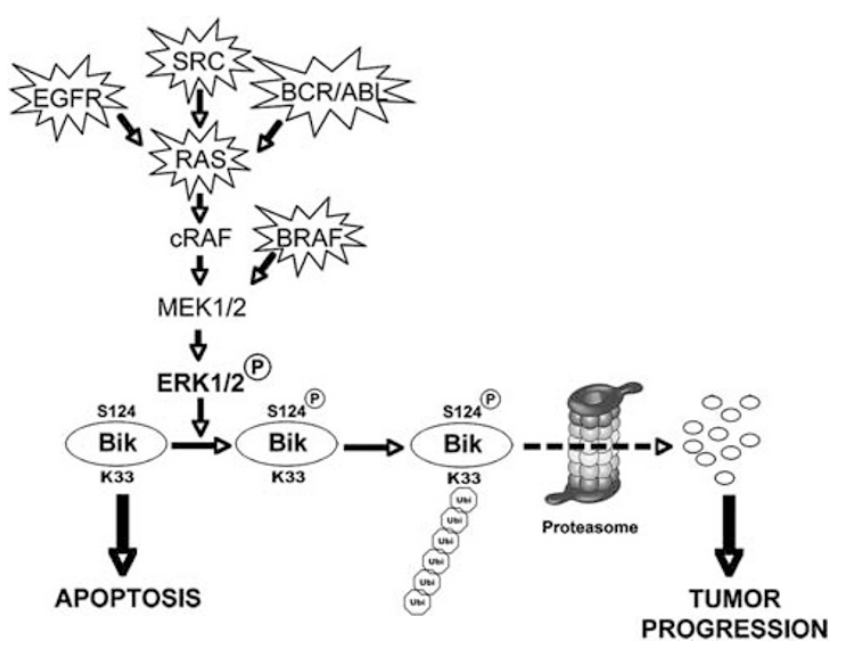

Figure 7 Bik concentration and sensitivity to apoptosis may be under Erk1/2 control in cancer cells. p-Erk1/2-dependent phosphorylation of Bik on residue 124 targets Bik for ubiquitylation, triggers its degradation by the proteasome and promotes cell survival. Bik silencing demonstrates that Bik downregulation has a crucial role in protecting cells with deregulated $p$-Erk $1 / 2$ against apoptosis, although other $\mathrm{Bcl}-2$ family proteins are also regulated by $p$-Erk1/2. Erk1/2 activation is the result of the deregulation of numerous oncogenes that are activated in many types of cancers. This suggests that the above-described mechanism of apoptosis control might be of general interest in cancer biology

(Souffelweyersheim, France): PD184352/Cl-1040 (10 $\mu \mathrm{M})$, dasatinib $(50 \mathrm{nM})$ and PLX-4720 (500 nM). ABT-737 and its inactive enantiomer A-793844 were obtained from Abbott Laboratories (Rungis, France) and used at $1 \mu \mathrm{M}$ (MTA 18968). These drugs were used overnight except when otherwise indicated. In half-life experiments, cycloheximide and actinomycin D were used at $50 \mu \mathrm{g} / \mathrm{ml}$ and $1 \mu \mathrm{M}$, respectively.

Western blotting and immunofluorescence. Antibodies used in western blotting are listed in Supplementary experimental procedures. Western blots were revealed by enhanced chemiluminescence (Immobilon, Millipore, Molsheim, France) using a ChemiDoc XRS system (Bio-Rad, Marnes-la-Coquette, France). Densitometric analysis was performed using QuantityOne software (v4.6.6, Bio-Rad). Images were collected using an Axioplan 2 microscope (Carl Zeiss, Le Pecq, France) equipped with $\times 40$ and $\times 100$ objectives.

Cell fractionation and mitochondria isolation. Mitochondria were prepared as already described. ${ }^{38}$ Briefly, cells were scraped, washed and broken (30 strokes) in cold MB buffer containing Complete protease inhibitor (Roche, Boulogne-Billancourt, France) with a 2-ml glass/glass homogenizer (Kontes, VWR). Homogenates were cleared at $1500 \times g$ and mitochondria were spun down at $10000 \times g$.

tBid-induced cyt-c release and Bax insertion in isolated mitochondria. This test was performed as previously described ${ }^{38}$ using caspase-8-cleaved Bid (tBid) kindly provided by J-C Martinou (Geneva, Switzerland). Bax insertion was assessed using alkali treatment. ${ }^{39}$

Pull-down experiments. Control and v-src-transformed NIH-3T3 cells were lysed in RIPA buffer containing complete protease inhibitor (Roche) and $1 \mathrm{mM}$ PMSF. To study Bik ubiquitylation, cells transfected with ubiquitin-myc (when indicated) and HA-Bik expression vectors were treated with MG132. Lysates were incubated with $10 \mu \mathrm{g}$ of either anti-myc or anti-HA antibodies for $16 \mathrm{~h}$ at $4{ }^{\circ} \mathrm{C}$. Then, $30 \mu \mathrm{l}$ of proteinA-Sepharose beads (Sigma, Saint Quentin-Fallavier, France) were added for $4 \mathrm{~h}$ at $4{ }^{\circ} \mathrm{C}$. Beads were recovered by centrifugation, washed four-time in RIPA buffer, resuspended in Laemmli buffer and analyzed by western blotting against ubiquitin, HA or Bik in a $8 \%$ SDS-PAGE gel.

In vitro kinase assay. 293T cells were co-transfected for $24 \mathrm{~h}$ with $\mathrm{HA}-\mathrm{Bik}$ and p35 caspase-inhibitor constructs (two 140-mm dishes per condition). Then, cells 
were treated overnight with bortezomib $(1 \mu \mathrm{M})$ and PD184352 $(10 \mu \mathrm{M})$ to allow Bik accumulation and inhibit endogenous phosphorylation. Cells were further lysed in PK NEBuffer $1 \mathrm{X}$ as recommended by New England Biolabs (Evry, France). HA-Bik was immunoprecipitated from lysates by overnight incubation with anti-HA antibody (1: 100). Immunocomplexes were captured using Dynabeads-Protein $G$ beads (Life Technologies, Cergy Pontoise, France) for $3 \mathrm{~h}$, beads were washed three times in PK NEBuffer $1 \mathrm{X}$ and incubated with $100 \mathrm{U}$ of $\mathrm{p}$-Erk2 (NEB), $2 \mathrm{mM} \mathrm{ATP} \mathrm{(NEB)} \mathrm{and}$ $400 \mu \mathrm{g} / \mathrm{ml}$ of HA-peptide (Sigma) for $1 \mathrm{~h}$ at $30^{\circ} \mathrm{C}$ under agitation. MBP5 protein (NEB) was used as a positive control $(100 \mu \mathrm{g} / \mathrm{ml})$. After magnetic separation, the supernatant was incubated with Laemmli buffer and analyzed by western blotting against phospho-Threonine (pAb from Invitrogen \#71-8200; $1: 200$ ).

\section{Conflict of Interest}

The authors declare no conflict of interest.

Acknowledgements. We thank Helene Akpolou, Anne Bénard and Valérie Sornin for technical support. We are grateful to Pr Jean-Claude Martinou (Geneva) for kindly providing caspase-8-cleaved Bid (tBid), recombinant Bax and for critical reading of this work. Dr. Safa Lucken-Ardjomande's critical assessment of our data was helpfu for establishing the $v$-src cellular model. We are grateful to Abbott for providing us with ABT-737 (MTA 18968). This work was partly supported by grants from 'La Ligue Contre le Cancer' (Comités de la Drôme et du Rhône) and by the Hospices Civils de Lyon - Groupement des Hôpitaux du Nord (Junior research grant 2009).

1. Martin GS. The hunting of the Src. Nat Rev Mol Cell Biol 2001; 2: 467-475

2. Takeya $T$, Hanafusa $H$. Structure and sequence of the cellular gene homologous to the RSV src gene and the mechanism for generating the transforming virus. Cell 1983; 32 881-890.

3. Talamonti MS, Roh MS, Curley SA, Gallick GE. Increase in activity and level of pp60c-src in progressive stages of human colorectal cancer. J Clin Invest 1993; 91: 53-60.

4. Zhang S, Huang W-C, Li P, Guo H, Poh S-B, Brady SW et al. Combating trastuzumab resistance by targeting SRC, a common node downstream of multiple resistance pathways. Nat Med 2011; 17: 461-469.

5. Evan Gl, Wyllie AH, Gilbert CS, Littlewood TD, Land $H$, Brooks $M$ et al. Induction of apoptosis in fibroblasts by c-myc protein. Cell 1992; 69: 119-128.

6. Clark W, Gillespie DA. Transformation by v-Jun prevents cell cycle exit and promotes apoptosis in the absence of serum growth factors. Cell Growth Differ 1997; 8: 371-380.

7. Ries S, Biederer C, Woods D, Shifman O, Shirasawa S, Sasazuki T et al. Opposing effects of Ras on p53: transcriptional activation of mdm2 and induction of p19ARF. Cell 2000; 103 : 321-330.

8. Shan B, Lee WH. Deregulated expression of E2F-1 induces S-phase entry and leads to apoptosis. Mol Cell Biol 1994; 14: 8166-8173.

9. Sovová V, Friis R, Fidlerová H, Hlozánek I. c-myc gene activation as a permanent trait of RSV-infected quail cells. Int J Cancer 1993; 53: 983-987.

10. Zindy F, Eischen CM, Randle DH, Kamijo T, Cleveland JL, Sherr CJ et al. Myc signaling via the ARF tumor suppressor regulates p53-dependent apoptosis and immortalization. Genes Dev 1998; 12: 2424-2433.

11. Pasteau S, Loiseau L, Brun G. Proliferation of chicken neuroretina cells induced by v-src, in vitro, depends on activation of the E2F transcription factor. Oncogene 1997; 15: 17-28.

12. Reginato MJ, Mills KR, Becker EBE, Lynch DK, Bonni A, Muthuswamy SK et al. Bim regulation of lumen formation in cultured mammary epithelial acini is targeted by oncogenes. Mol Cell Biol 2005; 25: 4591-4601.

13. Gillet G, Guerin M, Trembleau A, Brun G. A Bcl-2-related gene is activated in avian cells transformed by the Rous sarcoma virus. EMBO J 1995; 14: 1372-1381.

14. Bonni A, Brunet A, West AE, Datta SR, Takasu MA, Greenberg ME. Cell survival promoted by the Ras-MAPK signaling pathway by transcription-dependent and -independent mechanisms. Science 1999; 286: 1358-1362.

15. Webb BL, Jimenez E, Martin GS. v-Src generates a p53-independent apoptotic signal. Mol Cell Biol 2000; 20: 9271-9280.

16. Johnson D, Agochiya M, Samejima K, Earnshaw W, Frame M, Wyke J. Regulation of both apoptosis and cell survival by the v-Src oncoprotein. Cell Death Differ 2000; 7: 685-696.
17. Yamaguchi $\mathrm{H}$, Wang $\mathrm{HG}$. The protein kinase PKB/Akt regulates cell survival and apoptosis by inhibiting Bax conformational change. Oncogene 2001; 20: 7779-7786.

18. Zha J, Harada H, Yang E, Jockel J, Korsmeyer SJ. Serine phosphorylation of death agonist $B A D$ in response to survival factor results in binding to 14-3-3 not BCL-X(L). Cell 1996; 87: 619-628.

19. Cardone MH, Roy N, Stennicke HR, Salvesen GS, Franke TF, Stanbridge E et al. Regulation of cell death protease caspase-9 by phosphorylation. Science 1998; 282: 1318-1321.

20. Alvarado-Kristensson $M$, Melander $F$, Leandersson $K$, Rönnstrand $L$, Wernstedt $C$, Andersson T. p38-MAPK signals survival by phosphorylation of caspase-8 and caspase-3 in human neutrophils. J Exp Med 2004; 199: 449-458.

21. Lucken-Ardjomande S, Montessuit S, Martinou J-C. Bax activation and stress-induced apoptosis delayed by the accumulation of cholesterol in mitochondrial membranes. Cell Death Differ 2008; 15: 484-493.

22. Oltersdorf T, Elmore S, Shoemaker A, Armstrong R, Augeri D, Belli B et al. An inhibitor of Bcl-2 family proteins induces regression of solid tumours. Nature 2005; 435: 677-681.

23. Chinnadurai G, Vijayalingam S, Rashmi R. BIK, the founding member of the BH3-only family proteins: mechanisms of cell death and role in cancer and pathogenic processes. Oncogene 2008; 27 (Suppl 1): S20-S29.

24. Mebratu YA, Dickey BF, Evans C, Tesfaigzi Y. The BH3-only protein Bik/Blk/Nbk inhibits nuclear translocation of activated ERK $1 / 2$ to mediate IFNgamma-induced cell death. $\mathrm{J} C e l l$ Biol 2008; 183: 429-439.

25. Solit DB, Garraway LA, Pratilas CA, Sawai A, Getz G, Basso A et al. BRAF mutation predicts sensitivity to MEK inhibition. Nature 2006; 439: 358-362.

26. Shirasawa S, Furuse M, Yokoyama N, Sasazuki T. Altered growth of human colon cancer cell lines disrupted at activated Ki-ras. Science 1993; 260: 85-88.

27. Gandhi J, Zhang J, Xie Y, Soh J, Shigematsu H, Zhang W et al. Alterations in genes of the EGFR signaling pathway and their relationship to EGFR tyrosine kinase inhibitor sensitivity in lung cancer cell lines. PLoS ONE 2009; 4: e4576.

28. Lopez-Crapez E, Chypre C, Saavedra J, Marchand J, Grenier J. Rapid and large-scale method to detect K-ras gene mutations in tumor samples. Clin Chem 1997; 43 (6 Part 1): 936-942.

29. Harada H, Quearry B, Ruiz-Vela A, Korsmeyer SJ. Survival factor-induced extracellular signal-regulated kinase phosphorylates BIM, inhibiting its association with BAX and proapoptotic activity. Proc Natl Acad Sci USA 2004; 101: 15313-15317.

30. Nguyen TK, Rahmani M, Harada $H$, Dent $P$, Grant S. MEK1/2 inhibitors sensitize $\mathrm{Bcr} / \mathrm{Abl}+$ human leukemia cells to the dual Abl/Src inhibitor BMS-354/825. Blood 2007; 109: 4006-4015.

31. Boyd J, Gallo G, Elangovan B, Houghton A, Malstrom S, Avery B et al. Bik, a novel death-inducing protein shares a distinct sequence motif with Bcl-2 family proteins and interacts with viral and cellular survival-promoting proteins. Oncogene 1995; 11 : 1921-1928.

32. Nikrad M, Johnson T, Puthalalath $H$, Coultas L, Adams J, Kraft AS. The proteasome inhibitor bortezomib sensitizes cells to killing by death receptor ligand TRAIL via BH3-only proteins Bik and Bim. Mol Cancer Therapeutics 2005; 4: 443-449.

33. Marshansky V, Wang X, Bertrand R, Luo H, Duguid W, Chinnadurai G et al. Proteasomes modulate balance among proapoptotic and antiapoptotic Bcl-2 family members and compromise functioning of the electron transport chain in leukemic cells. $J$ Immuno 2001; 166: 3130-3142.

34. Zhu H, Zhang L, Dong F, Guo W, Wu S, Teraishi F et al. Bik/NBK accumulation correlates with apoptosis-induction by bortezomib (PS-341, Velcade) and other proteasome inhibitors. Oncogene 2005; 24: 4993-4999.

35. Luciano F, Jacquel A, Colosetti P, Herrant M, Cagnol S, Pages G et al. Phosphorylation of Bim-EL by Erk1/2 on serine 69 promotes its degradation via the proteasome pathway and regulates its proapoptotic function. Oncogene 2003; 22: 6785-6793.

36. Weinstein IB, Joe A. Oncogene addiction. Cancer Res 2008; 68: 3077-3080; discussion 3080.

37. Néel B, Lopez J, Chabadel A, Gillet G. Lithium suppresses motility and invasivity of $v$-src-transformed cells by glutathione-dependent activation of phosphotyrosine phosphatases. Oncogene 2009; 28: 3246-3260.

38. Eskes R, Antonsson B, Osen-Sand A, Montessuit S, Richter C, Sadoul R et al. Baxinduced cytochrome $C$ release from mitochondria is independent of the permeability transition pore but highly dependent on Mg2+ ions. J Cell Biol 1998; 143: 217-224.

39. Fujiki Y, Hubbard AL, Fowler S, Lazarow PB. Isolation of intracellular membranes by means of sodium carbonate treatment: application to endoplasmic reticulum. J Cell Bio 1982; 93: 97-102.

\section{Supplementary Information accompanies the paper on Cell Death and Differentiation website (http://www.nature.com/cdd)}

